\title{
Operative Therapie der Varikose
}

\author{
Kornelia Böhler
}

Eingegangen: 30. März 2016 / Angenommen: 9. Juni 2016 / Online publiziert: 12. Juli 2016

(c) Der/die Autor(en) 2016. Dieser Artikel ist eine Open-Access-Publikation.

\begin{abstract}
Zusammenfassung Der Chirurgie der V saphena magna zugrunde liegt das theoretische Konzept des Trendelenburgschen Privatkreislaufes (1891). Als logische Konsequenz ergab sich, dass nur die Unterbrechung des Kreislaufs bzw. die Resektion refluxiver Venen zu einem therapeutischen Erfolg führen kann. Über die Jahre wurde das chirurgische Verfahren des Venenstrippings modifiziert und $\mathrm{zu}$ seiner effektivsten Form, der Crossectomie mit angeschlossenem Stripping weiterentwickelt. Jahrzehntelang galten Crossectomie und Stripping als Goldstandard in der chirurgischen Therapie der Varikose, an deren Mitte- und Langzeitergebnissen alle später entwickelten minimal invasiven Therapieverfahren gemessen wurden. Dank des technischen Fortschrittes wurde die klassische Chirurgie der Varizen, zumindest in den westlichen Industrieländern, weitgehend von minimal traumatisierenden, hocheffektiven Therapieverfahren abgelöst. Für eine kleine Gruppe von Patienten mit speziellen anatomischen Verhältnissen und in Regionen mit geringen Gesundheitsresourcen bleibt das Stripping weiterhin ein therapeutisches Standardverfahren.
\end{abstract}

Schlüsselwörter Crossectomie - Stripping · Miniphlebectomie $\cdot$ CHIVA

\section{Surgery of varicose vein insufficiency}

Summary The basis of surgery of great saphenous vein insufficiency is the concept of "privat circulation" coined by Trendelenburg in 1891. It was only logical

\section{Ao. Univ.-Prof. Dr. med. univ. K. Böhler ( $₫)$}

Universitätsklinik für Dermatologie, Medizinische

Universität Wien, Währinger Gürtel 18-20, 1090 Wien,

Österreich

E-Mail: kornelia.boehler@meduniwien.ac.at that the dissection of the insuffcient vein or it's partial or complete resection could bring about healing. Over the years varicose vein stripping was modified to finally result in the highly effectiv concept of crossectomy and stripping. For decades this was the Goldstandard in surgery of varicose vein insufficiency. All the other minimally invasive therapeutic techniques which have been developed in the last decades had to compete with crossectomy and stripping. Thanks to progress in technical development the classic stripping procedure has been replaced by highly effectiv, minimally invasiv procedures, at least in the western industrialisied countries. For a minority of patients with specific anatomical pathologies as well as countries with limited health resources vein stripping remains a surgical standard procedure.

Keywords Crossectomy · Stripping · Phlebectomie · CHIVA

\section{Einleitung}

Die Indikation für eine operative Therapie der Varikose besteht bei allen Ausprägungsgraden der Chronisch Venösen Insuffizienz (CVI), also ab einem Stadium C3, aber auch bei einer ausgeprägten Varikose des Stadiums C2, wenn der Patient noch klinisch beschwerdefrei ist. Das therapeutische Prinzip die gestörte venöse Hämodynamik durch chirurgische Beseitigung refluxiver Venen zu korrigieren datiert zurück ins letzte Jahrtausend als William Wayne Babcock den Venenextraktor erfand. Bis in die 90er Jahre des letzten Jahrtausends waren Crossectomie und Stripping der Gold Standard der chirurgischen Therapie der Varikose. Heute setzen sich zunehmend endoluminale thermische oder chemische Verfahren durch, da sie bei gleichwertiger Effektivität die postoperative Morbidität des Patienten deutlich senken [1]. 


\section{Crossectomie und Stripping}

Unter einer Crossektomie versteht man die Ligatur der oberflächlichen Stammvene (Vena saphena magna oder parva) (VSM,VSP) an der Einmündung in die $\mathrm{V}$ femoralis communis bzw. V politea. Dabei werden alle Seitenäste im Mündungsbereich unterbunden. Für die VSM Crosse fordern manche Chirurgen eine zirkuläre Freilegung der $\mathrm{V}$ femoralis über $2-3 \mathrm{~cm}$ distal und proximal der Mündung [2]. Andere sehen ein derart radikales Vorgehen als übertrieben an, vor allem in Anbetracht der Kenntnis, dass auch radikaler Op Techniken das Problem des Crosserezidivs nicht verhindern können. Auch bei bündiger Ligatur der VSM mit Resektion des Magnastumpfes (flush ligation) mit oder ohne Endothelinversion bleibt das Verfahren Rezidiv anfällig [3]. Im Bereich der V saphena parva wird auf Grund der komplexen anatomischen Verhältnisse das Prinzip der flush ligation häufig zu Gunsten einer lediglich mündungsnahem Ligatur verlassen. Verletzungen des $\mathrm{N}$ peronaeus, die bei $2-4 \%$ der Behandelten vorkommen, können so weitgehend vermieden werden. Bedenkt man, dass endoluminale Verfahren, die systematisch auf die Crossektomie verzichten, mittelfristig die gleichen Erfolgsraten aufweisen wie das klassische Stripping, drängt sich der Verdacht auf, dass die Bedeutung der Crossektomie überschätzt wurde [4]. Unangefochten ist hingegen der Stellenwert der Entfernung erkrankter Stammvenenabschnitte. Da mehrfach gezeigt wurde, dass das Rezidivrisiko nach Crossektomie mehr als doppelt so hoch ist als nach Crossektomie und Stripping wurde der Weg Stammvenen erhaltend zu operieren wieder verlassen. Funktionell ausreichend ist ein VSM Stripping bis auf Kniehöhe, dabei wird die Rate der N. saphenus Läsionen auf 7 \% gesenkt. Für die VSP besteht keine klare Evidenz, dass das Gefäßstripping mit einer geringeren Rezidivrate behaftet ist. Allerdings wird eine niedrige Rezidivrate mit einem bis $\mathrm{zu} 20 \%$ igen Risiko der N suralis Verletzung erkauft. Major Komplikationen, in erster Linie Verletzungen der tiefen Gefäße, sind mit 0,8\% beziffert und treten vor allem bei Recrossektomien auf. Ungelöst bleibt trotz jahrzehntelanger Anwendung mehr oder weniger radikaler, klassischer chirurgischer Verfahren das Problem der Rezidivvarikose.

\section{Rezidvvarikose nach Stripping}

Der ewige Streit in der wissenschaftlichen Community, ob Rezidive mehrheitlich auf technische Fehler, bei mangelnder chirurgischer Kompetenz, oder auf eine konstitutionelle Neigung zur Gefäßneubildung zurückzuführen ist, ist nicht entschieden. So konnte an einem Patientenkollektiv, das über 32 Jahre verfolgt wurde gezeigt werden, dass nach VSM Stripping $60 \%$ der Patienten Rezidivvarizen aufwiesen, davon waren $70 \%$ ausgehend von der Crosseregion [5]. $\mathrm{Zu}$ etwa gleichen Teilen war das Rezidiv auf ein singuläres
Rezidivgefäße oder eine ungeordnete Gefäßneubildung (Neovascularisation) im Bereich der ehemaligen Cosse oder ein Crosse fernes Gefäß zurückzuführen. Die klinische Bedeutung der Neovascularistion bleibt umstritten, da klinisch relevante Rezidive eher auf operationstechnische Fehler mit Belassung axialer Seitenäste, auf strategische Fehler mit Belassung der VSM oder auf singuläre Crosserezidivgefäße als auf eine Neovaskularisation zurückzuführen sind [6]. Die Reexploration der saphenofemoralen Mündungsregion kann technisch schwierig sein. Sie erfolgt in der Regel über eine laterale oder mediale Freilegung der $\mathrm{V}$ femoralis, ist zeitaufwendig und mit einer hohen Komplikationsrate behaftet. Da auch radikale Recrossektomien und das Einbringen von Barrieren ein neuerliches Rezidiv nicht verhindern können, werden zunehmend minimal invasive Techniken bevorzugt, die in erster Linie auf eine Sanierung des Varizenreservoirs abzielen [7].Bewährt haben sich die Miniphlebektomie und die Sklerosierungstherapie.

\section{Miniphlebektomie}

Unter Miniphlebektomie versteht man die chirurgische Entfernung von retikulären Varizen unter Wahrung höchster ästhetischer Ansprüche. Nach adäquater Markierung im Stehen werden die Varizen mit speziellen hakenförmigen Instrumenten aus dem Subcutanraum hervorgezogen, mit spatelförmigen Instrumenten mobilisiert und extrahiert. Die Wunden werden lediglich mit Steristrips verschlossen.

\section{CHIVA}

CHIVA ist das Acronym für „Cure conservatrice et hemodynamique de l'insuffisance veineuse en ambulatoire“. 1988 erstmals von Claude Franceschi beschrieben beruht dieses Therapiekonzept auf der gezielten Unterbrechung pathologischer Rezirkulationskreisläufe bei gleichzeitigem Erhalt der Wiedereintrittswege des retrograden Blutvolumens in das tiefe Venensystem und Beseitigung nicht drainierter oberflächlicher Varizen [8]. Die Methode ist vor allem im französischen Sprachraum verbreitet. Franceschi geht aus von einer hierarchischen Ordnung der Venen entsprechend ihrer Topographie: R1 die subfaszial gelegenen Leitvenen und Muskelvenen, R2 die zwischen der Muskelfaszie und der Saphenafaszie gelegenen Stammvenen und anteilig die V saphena accessoria lateralis und medialis, R3 die in der Subcutis gelegenen Seitenäste und retikulären Varizen, R4 subcutane Venen die zwei R2 Venen miteinander verbinden. Franceschi unterscheidet zwischen geschlossenen Shunts mit direkter Rezirkulation des Blutvolumens. Klassisches Beispiel ist die inkompetente saphenofemorale Mündung, bei der die Rezirkulation der insuffizienten Stammvene über eine Perforansvene zurück in die tiefe Venen erfolgt (Shunttyp I : R1-R2-R1). Ist in den Shunt zusätzlich ein R3 Seitenast involviert, 
spricht man von einem Shunttyp 1+R3. Beim Shunttyp II (R2-R3-R2) finden sich Seitenastvarizen, aber keine unmittelbare Verbindung zum tiefen Venensystem. Dieser Typ ist selten. Beim Shunttyp 3 (R1-R2-R3R1) erfolgt die Rezirkulation aus der Stammvene über Seitenäste direkt, unter Umgehung der Stammvenen, ins tiefe Venensystem. Zur Sanierung des Shunttyps I muss der oberste Refluxpunkt, beispielsweise die saphenofemorale Mündung, unterbrochen werden. Durch Ausschaltung des Refluxvolumens kommt es zur Rückstellung der Stammvene, die Klappen werden wieder schlussfähig. Beim Typ1+R3 werden zusätzlich die Seitenäste an ihrem Abgang aus der insuffizienten Stammvene ligiert. Am distalen Insuffizienzpunkt der insuffizienten Stammvene bleibt die Rezirkulationsperforante erhalten. Beim Typ3 wird im ersten Schritt der insuffiziente Seitenast an der Stammvene ligiert, reicht diese Intervention nicht aus um einen orthograden Fluss in der Stammvene zu erzielen, wird in einem zweiten Schritt eine Stammvenenligatur am oberen Insuffizienzpunkt durchgeführt. Die CHIVA Methode erfordert eine genaue Kenntnis der venösen Hämodynamik am erkrankten Bein, eingeleitet durch eine exakte duplexsonographische Abklärung präoperativ. Ein kürzlich publizierter Cochrane Review kommt zu dem Ergebnis, dass CHIVA die Rate der Rezidivvarizen reduziert, bei gleichzeitig geringer Komplikationsrate. Einschränkend wird allerdings festgestellt, dass die Zahl verwertbarer Studien gering ist [9].

\section{Chirurgie der Perforansvenen}

Die Bedeutung insuffizienter Perforansvenen (IP) im Rahmen der CVI ist umstritten. Allgemein anerkannt ist, dass die Anzahl, der Durchmesser und das Refluxvolumen von Perforansvenen mit dem Grad der venösen Insuffizienz korreliert [10]. Weitgehende Übereinstimmung herrscht darüber , dass Perforansvenen mit einem subfaszialen Durchmesser $>3,9 \mathrm{~mm}$ und einem Reflux >350-500 ms als insuffizient anzusehen sind. Die Bedeutung IP, vor allem im Bereich des medialen Unterschenkels, steigt mit dem Grad der CVI, und ist vor allem für die Stadien C5 und C6 untersucht. Auf Grund der mangelhaften Datenlage kann derzeit nicht festgestellt werden, inwieweit die Ausschaltung IP für die Therapie der CVI entscheidend ist $[11,12]$. Wichtigster Bias ist, dass IP meist gemeinsam mit insuffizienten Stammvenen operiert werden. Die Unterbrechung des axialen Refluxes hat hämodynamisch den größeren Stellenwert. Darüber hinaus wissen wir, dass nach der Sanierung epifaszialer axialer Refluxe IP wieder kompetent werden können. In einer unkritischen Überschätzung IP wurden höchst traumatisierende operative Verfahren angewandt wie beispielsweise die Linton'sche Operation mit longitudinaler Eröffnung des Unterschenkels entlang der Linton'schen Linie, in dem Bestreben alle Perforansvenen $\mathrm{zu}$ eradizieren und so den venösen
Druck auf die kutane Zirkulation zu senken. Erhebliche Wundheilungsstörungen waren die Folge. Auch weniger ausgedehnte transkutane Zugänge können bei fortgeschrittenen Hautveränderungen im Unterschenkelbereich zu Wundheilungsstörungen führen. 1985 wurde von Hauer die subfasziale endoskopische Perforansdissektion (SEPS) eingeführt [13]. Mit dieser Methode gelang es über einen transkutanen Zugang im proximalen Unterschenkelbereich, somit immer via intakter Haut, die IP subfaszial zu koagulieren und durchtrennen. In der Langzeitbeobachtung zeigten sich allerdings residuelle Perforantes in bis $\mathrm{zu}$ $20 \%$ der Behandelten. In einer duplexgestützten eigenen Untersuchung stellten wir bei $20 \%$ der nach 3,7 a nachuntersuchten SEPS Patienten neu aufgetretene Perforantes fest [14]. Die endoskopische Methode ist apparativ aufwendig und hat eine flache Lernkurve.

Entsprechend adaptierte Katheter führen dazu, dass sich endoluminale thermische Verfahren $\mathrm{zu}^{-}$ nehmend auch in der Behandlung IP etablieren. Die Kurzzeitverschlussraten werden zwischen $60 \%$ und $90 \%$ angegeben wobei wieder eine deutliche Assoziation zwischen der Erfahrung des Operateurs und der Effizienz der Methode besteht. Entsprechende Lernkurven müssen somit eingerechnet werden.

Open access funding provided by Medical University of Vienna.

Interessenkonflikt K. Böhler gibt an, dass kein Interessenkonflikt besteht.

Open Access Dieser Artikel unterliegt den Bedingungen der Creative Commons Attribution Noncommercial License. Dadurch sind die nichtkommerzielle Nutzung, Verteilung und Reproduktion erlaubt, sofern der/die Originalautor/en und die Quelle angegeben sind.

\section{Literatur}

1. Nesbitt C, Bedenis R, Bhattacharya V, et al. Endovenous ablation (radiofrequency andlaser) andfoam sclerotherapy versus open surgery for great saphenous vein varices. Cochrane Database Syst Rev. 2014;7:CD005624.

2. Stenger D, Hartmann M. High ligation and vein stripping. The classic procedure. Hautarzt. 2012;63(8):616-21.

3. Heim D, Negri M, Schlegel U, et al. Resecting the great saphenous stump with endothelial inversion decreases neither neovascularisation nor thigh varicosity recurrence. JVasc Surg. 2008;47(5):1028-32.

4. Rasmussen L, Lawaetz M, Bjoern L, et al. Randomized clinical trial comparing endovenous laser ablation and stripping of the great saphenous vein with clinical and duplex outcome after 5 years. JVasc Surg. 2013;58(2):421-6.

5. Fischer R, ChandlerJ, DeMaeseneerM,etal. Theunresolved problem of recurrent saphenofemoral reflux. JAm Coll Surg. 2002;195:80-94.

6. Egan B, Donnelly M, Bresnihan M, etal. Neovascularisation: an innocent bystander in recurrent varicose veins. J Vasc Surg. 2006;44(6):1279-84.

7. Pittaluga P, Chastanet S, Locret T, et al. Retrospective evaluation of the need of a redo surgery at the groin 


\section{themenschwerpunkt}

for the surgical treatment of varicose vein. J Vasc Surg. 2010;51:1442-50

8. Franceschi C. Ambulatory and hemodynamic treatment of venous insufficiency (CHIVA cure). J Mal Vasc. 1992;17(4):291-300.

9. Bellmunt-Montoya S, Escribano JM, Dime J, et al. CHIVA method for the treatment of varicose veins. Cochrane Database Syst Rev. 2013;7:CD009648.

10. Stuart WP, Donald JA, Allan PL, et al. The relationship between the number, competence and diameter of medial calf perforating veins and the clinical status in healthy subjects and patients with lower limb venous disease. JVasc Surg. 2000;32:138-43.
11. O'Donnell TF Jr. Part two:against the motion.Venous perforator surgery is unproven and does not reduce recurrences. EurJVasc Endovasc Surg. 2014;48:242-6.

12. Whiteley MS. Part one: for the motion. Venous perforator surgery is proven and does reduce recurrences. Eur J Vasc Endovasc Surg. 2014;48:239-42.

13. Hauer G. Endoscopic subfascial dissection of perforating veins-preliminary report. Vasa. 1985;14:59-61.

14. Roka F, Binder M, Boehler-Sommeregger K. Mid term recurrence rate of incompetent perforating veins after combined superficial vein surgery and subfascial endoscopic perforating vein surgery. JVasc Surg. 2006;44(2):359-63. 